\title{
Cost-effectiveness Assessment of 5G Systems with Cooperative Radio Resource Sharing
}

\author{
Vladimir Nikolikj, Member, IEEE, and Toni Janevski, Senior Member, IEEE
}

\begin{abstract}
By use of techno-economic analysis of heterogeneous hierarchical cell structures and spectral efficiencies of the forthcoming advanced radio access technologies, this paper proposes various cost-efficient capacity enlargement strategies evaluated through the level of the production cost per transferred data unit and achievable profit margins. For the purpose of maximizing the aggregate performance (capacity or profit), we also assess the cooperative manners of radio resource sharing between mobile network operators, especially in the cases of capacity over-provisioning, when we also determine the principles to provide guaranteed data rates to a particular number of users. The results show that, for heavily loaded office environments, the future $5 \mathrm{G}$ pico base stations could be a preferable deployment solution. Also, we confirm that the radio resource management method with dynamic resource allocation can significantly improve the capacity of two comparably loaded operators which share the resources and aim to increase their cost effectiveness.
\end{abstract}

Keywords - 5G millimeter wave, Cost-effectiveness, EBIT margin, Guaranteed QoS, IEEE 802.11ad, LTE-Advanced, Radio Resource Management, Radio Resource Sharing.

\section{INTRODUCTION}

$\mathrm{T}^{\mathrm{s}}$ HE lack of microwave spectrum to be used by today's and cellular systems from the recent future enabled with radio access technologies (RATs) like 4G LTE Advanced or LTE-A (LTE Release 10 and beyond) [1], forced the recent publications to focus extensively on the coverage and capacity performance of the so called millimeter wave (mmW) systems, utilizing the huge amount of the available spectrum in the $\mathrm{mmW}$ bands $(30$ $\mathrm{GHz}$ - $300 \mathrm{GHz}$ ) [2]. The capacity and user data rate performances measured in multi-gigabits per second, as outlined in [3]-[6], were our exact motivation, to evaluate the financial ability of new mmW cellular systems (or 5G) to bridge the continuous "revenue gap". This research presents methodologies for the $5 \mathrm{G}$ systems mainly through delivering a variety of measurement results that show how e.g. 28, 38 and $73 \mathrm{GHz}$ carriers could be implemented with a bandwidth channel of up to $1 \mathrm{GHz}$. With this huge

Paper received April 9, 2015; revised July 1, 2015; accepted July 15, 2015. Date of publication November 15, 2015. The associate editor coordinating the review of this manuscript and approving it for publication was Prof. Aleksandar Nešković.

This paper is a revised and expanded version of the paper presented at the 22th Telecommunications Forum TELFOR 2014.

Vladimir Nikolikj is a Ph.D. candidate at the Faculty of Electrical Engineering and Information Technologies, Ss. Cyril and Methodius University, Skopje, Macedonia (e-mail: v.nikolikj@vipoperator.mk).

Toni Janevski, Ph.D. is a full professor at the Faculty of Electrical Engineering and Information Technologies, Ss. Cyril and Methodius University, Skopje, R. Macedonia (e-mail: tonij@feit.ukim.edu.mk). amount of available bandwidth, it is highly expected that capacity overprovisioning will occur often in many $5 \mathrm{G}$ based Advanced Wireless Access Heterogeneous Network (AWA-HetNet) layouts to be deployed in the future. Consequently, we analyze possible manners to exploit such overprovision and to propose a solution for an adequate quality of service (QoS) delivered in the form of a guaranteed data rate per individual user. Also, we assess the possibility of further capacity increases by the application of Radio Resource Management (RRM) methods in case of cooperative sharing of the radio resources among two mobile network operators (MNOs).

Capacity-cost comparisons of macro (MaBS), micro (MiBS), pico (PBS) HSPA base station (BS) sites, including IEEE $802.11 \mathrm{a} / \mathrm{n}$ access points (APs), are provided in [7], [8]. Cost analysis of LTE with HSPA deployed MaBS networks and femto (FBS) solutions are extensively covered in [9], [10]. Additionally, the profitability assessment is provided by various deployments of FBS and MaBS for HSPA and LTE mobile broadband (MBB) services in [11]-[13]. In all studies [7] - [13], authors perform their analysis in the frequency bands higher than $800 \mathrm{MHz}$ band and lower than 2.6 GHz. Various RRM functionalities for cooperative networks were outlined in [14], [15].

In our contribution, we originally present the comparative cost-capacity modeling, production cost calculation and business profitability evaluation of the $5 \mathrm{G}$ $\mathrm{mmW}$ systems integrated in the future AWA-HetNets together with the 4G LTE-A RAT and advanced Wi-Fi deployment like IEEE 802.11ad [2]. Considering the "up to date" initial and running cost drivers, we deliver results applicable to assess the profit margins of the various outdoor and indoor heavily-loaded AWA-HetNets. Also, we evaluate the suitable radio resource sharing in $5 \mathrm{G}$ based AWA-HetNets that will cost-effectively improve the capacity figures due to cooperation of the MNOs aiming to ensure data rate guarantees to end users.

The rest of this article is organized as follows. We describe the analysis approach through elaboration of specific coverage, capacity, and unit cost estimates for various BS and AP classes. Then we model the production cost in order to assess the profit margins of the outdoor deployed beyond $4 \mathrm{G}$ and $5 \mathrm{G}$ mobile systems. Further, we elaborate the investment case study focusing on the coverage, cost-capacity and profitability performances of the RATs within the newly built office area. Then, in Section VI we assess manners for sharing the radio resources between two MNOs with dynamic data loads in case of capacity overprovisioning with the indoor $5 \mathrm{G}$ pico 
base stations. Conclusions are drawn in Section VII.

\section{COVERAGE AND CAPACITY MODELING}

For modeling purposes, we approximate hexagonal cellular structures with a circle, with radius $r$, and we assume that subscribers are uniformly distributed within a cell. A BS of class $i$ is characterised by cell range $(\mathrm{km})$ related to coverage $\left(\mathrm{km}^{2}\right)$. Consequently, we dimension the BS site coverage " $A$ " as the circle area $\left(A=\pi r^{2}\right)$. According to [9], [10] and [16] we consider $0.57 \mathrm{~km}$ range for the three sectors 4G LTE-A urban MaBS. Based on the elaborations in [3], [6], we estimate $0.1 \mathrm{~km}$ range for 3sector 5G MMW metro bases stations (MetBS), deployed according to the 3GPP Urban Micro (UMi) model [17]. In line with [7], [18] and [19], for the indoor 3-sector 5G MMW PBS we consider a range of $0.02 \mathrm{~km}$. According to [12], we model the aggregated capacity of the system, $T_{\text {syst }}$, as follows:

$$
T_{\text {syst }}=W \cdot N_{\text {site }} \cdot N_{\text {cell }} \cdot S_{\text {eff }}
$$

where $W$ is an allocated bandwidth in $\mathrm{MHz}, N_{\text {site }}$ is the total number of BS sites within the system coverage area, $N_{\text {cell }}$ is the number of cells per BS site and $S_{\text {eff }}$ is the average cell spectral efficiency in $\mathrm{bps} / \mathrm{Hz} /$ cell. Based on [17] the average spectral efficiency for LTE-A varies from $3.8,4.2$ to $6.6 \mathrm{bps} / \mathrm{Hz} /$ cell. In line with [3], [6] for the $5 \mathrm{G}$ $\mathrm{mmW}$ system we consider an average cell spectral efficiency of $2.83 \mathrm{bps} / \mathrm{Hz} / \mathrm{cell}$. We consider LTE-A RAT with bandwidth chunks of $20 \mathrm{MHz}, \mathrm{mmW}$ system with $500 \mathrm{MHz}$ [3], [6] and [18]. For Wi-Fi, we consider 50$60 \%$ of the nominal bit rate of the underlying physical layer of [20]. Frame aggregations techniques are used to improve the Medium Access Control (MAC) layer efficiency [21]. According to [22], IEEE 802.11ad aims to provide cell capacity of $6756 \mathrm{Mbps}$ using OFDM and $2160 \mathrm{MHz}$ channel bandwidth at $60 \mathrm{GHz}$ unlicensed band, with a coverage range of $10 \mathrm{~m}$.

\section{COST DRIVERS ASSESSMENT}

We base our cost structure modelling on the methodology developed in [7, 11] by limiting the capital expenditures (CAPEX) and operational expenditures (OPEX) of the radio access network (RAN). The cost per item of type $i$ in present value, according to [8] is based on the standard method for cumulated discounted cash flows represented by summing up the total discounted annual expenditures for the whole network life cycle ( $\mathrm{K}$ years). The total cost of AWA-HetNets can be modeled as:

$$
C_{T O T}=N_{i} \sum_{i \in \Phi} \sum_{k=0}^{K-1} \frac{\alpha_{k, i}}{(1+\beta)^{k}}
$$

where $\alpha_{k, i}$ is the sum of expenditures, in terms of CAPEX and OPEX occurred within year $k$ of an BS/AP of type $i, \beta$ is the discount rate, $N_{i}$ is the number of BSs/APs that would be required of that kind, and $\Phi$ is the set of available BS/AP configurations. In line with [11], we use the weighted average cost of capital (WACC) value of $12.5 \%$ for $\beta$. We consider $20 \mathrm{k} €$ for a single carrier 3sector MaBS costs [7]. We assume $60 \mathrm{k} €$ for 3 -sector $4 \mathrm{G}$ MaBS with LTE-A RAT supporting 5 carriers [9].
Consequently, in line with [13] the total CAPEX for MaBS is $110 \mathrm{k} €$.

TABLE 1: COVERAGE, CAPACITY AND COST ESTIMATES FOR BSS/APS OF DIFFERENT RATS AND CELL TYPES WITH 1 CARRIER

\begin{tabular}{l|c|c|c|c}
\hline $\begin{array}{l}\text { BS Class/ } \\
\text { Parameter }\end{array}$ & $\boldsymbol{M a B S}$ & $\boldsymbol{M e t} \boldsymbol{B S}$ & $\boldsymbol{P B S}$ & $\begin{array}{c}\boldsymbol{W i}-\boldsymbol{F i} \\
\boldsymbol{A P}\end{array}$ \\
\hline RAT & $4 \mathrm{G}$ & $5 \mathrm{G}$ & $5 \mathrm{G}$ & IEEE \\
& LTE-A & $\mathrm{mmW}$ & $\mathrm{mmW}$ & $802.11 \mathrm{ad}$ \\
\hline Environment & Outd. & Outd. & Indo. & Indo. \\
Range (km) & 0.57 & 0.10 & 0.02 & 0.01 \\
Coverage (km²) & 1.02 & 0.03 & 0.001 & 0.0003 \\
Sectors & 3 & 3 & 3 & 1 \\
Bandwidth (MHz) & 20 & 500 & 500 & 2160 \\
Av. Cell SE (bps/Hz) & 3.8 & 2.83 & 2.83 & 3.13 \\
Av. Cell Capac. (Mbps) & 76 & 1415 & 1415 & 6756 \\
Av. Site Capac. (Mbps) & 228 & 4245 & 4245 & 6756 \\
CAPEX (k€) & 110 & 31 & 19 & 5 \\
Annual OPEX (k€) & 30 & 19 & 15 & 3.5 \\
Tot. discount. cost (k€) & 272.5 & 135.5 & 106.5 & 24.8 \\
\hline
\end{tabular}

For the reuse of the existing MaBS in line with [9] we consider $20 \mathrm{k} €$ related to site upgrade $(10 \mathrm{k} €)$ and to the additional radio equipment (10 k€). Regarding the MaBS annual OPEX we consider in total $30 \mathrm{k} €$. We assume OPEX of $10 \mathrm{k} €$ per year when an existing site is re-used. We model the related costs of MetBS and PBS, based on MiBS and PBS relations to MaBS from [7]. Regarding the Wi-FI APs, for the enterprise solutions we consider WLAN carrier grade access. The author of [7] outlines that the carrier grade AP is 10 times more expensive than WLAN AP for consumers, and that the cost for router and access getaway is $20 \mathrm{k} €$.

Due to the very high peak data rate expected with IEEE 802.11ad, we assume that carrier grade access point supporting IEEE $802.11 \mathrm{ad}$ will cost around $2.5 \mathrm{k} €$, and additional $2.5 \mathrm{k} €$ should be added per AP, assuming that the control equipment is divided between no more than 8 APs. Consequently, Table 1 summarizes the CAPEX, OPEX and total discounted cost estimated for 10 years. The resulting discounted cost structure of future AWAHetNets is given in Fig. 1, and grouped by radio, site and transmission related costs. As is seen, while radio and site costs dominate for MaBS, transmission costs are also significant for $5 \mathrm{G} \mathrm{mmW}$ MetBS and $5 \mathrm{G} \mathrm{mmW}$ PBS sites for optical high-speed backhaul.

\section{DEMAND ImPaCt ON PROFITABILITY OF THE FUtURE DENSE URBAN AWA-HETNET}

Our primary goal is to relate the total investment costs to the production costs and revenues in order to assess the business profitability of the AWA-HetNet using: a) $4 \mathrm{G}$ MaBS with LTE-A RAT and available $40 \mathrm{MHz}$ of bandwidth $(10 \mathrm{MHz}$ in $700 \mathrm{MHz}, 10 \mathrm{MHz}$ in $800 \mathrm{MHz}$ and $20 \mathrm{MHz}$ in $2.6 \mathrm{GHz}$ band) and b) using the 5G MetBS $\mathrm{mmW}$ system in $28 \mathrm{GHz}$ band. According to [23], [24], we consider low, moderate, and high demand levels of year 2020, or: 30, 120 and $500 \mathrm{~GB} /$ user/month. Further, we consider the dense urban area of $1.0 \mathrm{~km}^{2}$. Today, the capital city of the Philippines, Manila, has the highest population density in the world with around $42,000 / \mathrm{km}^{2}$ [25]. Nevertheless, we decide to consider a rather extremely populated area from the future (more than 6 times higher) with 250,000 citizens per $\mathrm{km}^{2}$ and mobile 
penetration rate of $80 \%$ in the market with 3 MNOs having equal share.

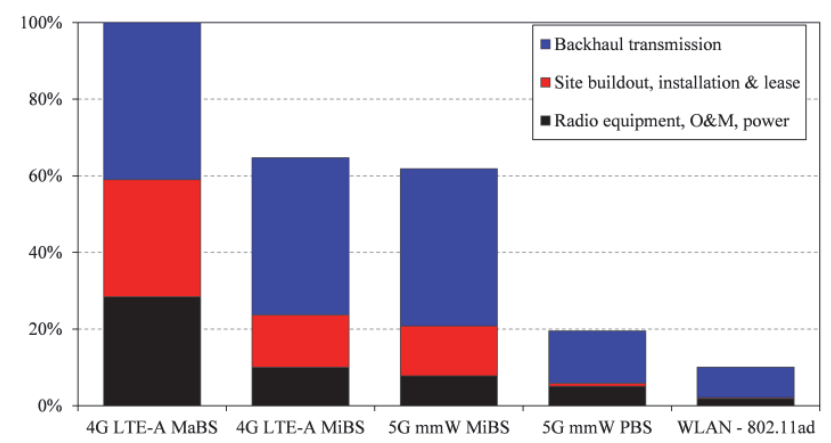

Fig. 1. BS discounted cost structure of AWA-HetNet.

We represent the generated traffic/capacity over a given area as a function of the MBB population density per MNO $\rho$ (or in our case 66,667 users $/ \mathrm{km}^{2}$ ) as follows [26]:

$$
G(t)=\rho \cdot \frac{1}{1024} \cdot 8 \frac{1}{f_{d h}} \cdot \frac{1}{n_{d}} \cdot \tau(t) \sum_{k} D_{k} \cdot s_{k}
$$

Here, $D_{k}$ represents the average data demand per month for terminal $k$ (i.e., $s_{p c}$, $s_{\text {tablet }}, s_{s . p h o n e}$ ), $\tau(t)$ represents a typical daily traffic variation in terms of percentage of the number of active users for a given time $t, f_{d h}$ denotes the number of hours of the day denoted as busy hours when the traffic is intensified and $n_{d}$ is the number of days of the month in which there is user activity. According to [24], we consider that the usage will be spread throughout 9 hours per day (translating into a busy hour rate of $11.11 \%$ ) or $f_{d h}=9$ across 30 days of a month or $n_{d}=30$. Further, in combination with the forecasted values, using (3) it is possible to calculate the peak area traffic demand at the busy hour as $G\left[\mathrm{Gbps} / \mathrm{km}^{2}\right]=\max _{t}(G(t))$ under the assumption that all of the subscribers are active during the busy/peak hour (i.e., $\underline{\tau}_{\max }=100 \%$ ), for the purpose of maximizing the probable cost-capacity estimates of an MNO. Consequently, Table 2 summarizes the area capacity values and the average user data rates, for each of the considered levels of user demand to be satisfied by the AWA-HetNet. The required CAPEX for the considered deployments is presented in the upper part of Table 3 . The calculations related to production costs and revenues that yield to the respective results in Table 3 are based on the approach described in [12] used to calculate the production cost per GB. To include the annualized CAPEX as a cost item in the results, the CAPEX for sites and radio equipment are depreciated over 20 and 5 years respectively, with WACC of $12.5 \%$. We assume average monthly revenue per user (ARPU) of $25.0 €$. The sum of the annualized CAPEX/GB and overall OPEX/GB (estimated as $45 \%$ of the overall revenues based on the assumption that $55 \%$ is the EBITDA earnings before interest, taxes, depreciation, and amortization) margin) yields the total production cost per GB. Finally, the profit (or the EBIT) margin is obtained as a ratio of the production cost per GB and revenue per GB. Fig. 2 depicts the resulting profit margin for each of the deployment scenarios considered. It can be seen that the smallest pressure on the profitability comes with a low usage, when the profit margin for all of the four deployments moves from $52 \%$ for the $4 \mathrm{G}$ LTE-A MaBS new sites, and up to around $54 \%$ for the case of $5 \mathrm{G} \mathrm{mmW}$ MetBS site reuse. Nevertheless, when maintaining higher demand levels, the profit margin of the MaBS greenfield deployment with $4 \mathrm{G}$ LTE-A drastically declines to $6 \%$. In the case of MaBS site reuse with $4 \mathrm{G}$ LTE-A, the profit margin is kept rather on the same level until the usage takes off to $500 \mathrm{~GB}$ per user and month when it is around 35\%. Also, with both deployments with MetBS $5 \mathrm{G} \mathrm{mmW}$ sites, the profit margin is maintained at the same level of around 54\% with no decline, showing the importance of having access with extremely high capacity compensating the coverage limitations.

\section{V.CAse Study - Complex of OfFice Buildings}

\section{A. Case Study Description}

In this section, we evaluate the cost-capacity performance of the future 5G MBB network in case when even higher (terabyte - TB) demand levels need to be maintained for the 25,000 indoor office users. Our particular interest is to assess the profit margin of deployments needed to satisfy the $36.0 \mathrm{~TB} / \mathrm{user} / \mathrm{month}$ called the "virtual reality office" demand, as defined in [24]. For that purpose we consider construction of a new office center in the $1.0 \mathrm{~km}^{2}$ urban indoor area. The office workers will be spread in the complex of 10, 5-floor buildings. We assess the deployment options with 4GLTA MaBS sites placed outside the buildings and 5G mmW PBS sites and WLAN IEEE 802.11 ad placed inside.

\section{B. Impact of Propagation and Penetration Losses}

Due to the increased demand for the 4G-LTA MaBS deployment, we assume that there are available $20 \mathrm{MHz}$ more in the $2.6 \mathrm{GHz}$ band or in total $60 \mathrm{MHz}$ in a downlink. In the deployment scenario with 4G-LTA MaBS we consider the potential increase of the number of 717 sites, needed only to satisfy the increased capacity demand within the considered complex of office buildings. Regarding the $5 \mathrm{G} \mathrm{mmW}$ MetBS deployment for indoor purposes, the authors of [3], [5] determined that their empirical results for the $100 \mathrm{~m}$ distance are mostly close to the Free-space loss mmW model as assumed in the Samsung study [18]. That model on top of the free space propagation assumes a correction factor of $20 \mathrm{~dB}$ and could be represented as follows:

$$
P L_{F S, d B}=112.4+20 \log _{10}\left(f_{c}\right)+20 \log _{10}(d)
$$

where, $f_{c}$ is the carrier frequency in $\mathrm{GHz}$, and $d$ is the distance in $\mathrm{km}$. For the $f_{c}=28 \mathrm{GHz}$, and $d=0.1 \mathrm{~km}$, we obtain that PLFS is $121.3 \mathrm{~dB}$. According to [4], in order to compensate for the $42.0 \mathrm{~dB}$ of additional wall attenuation, out of (4) we calculate that an additional extremely high number of 4,000 $\mathrm{mmW}$ MetBS sites have to be deployed.

TABLE 2: TARGETED USER DATA RATES (MBPS) AND ESTIMATED AREA TRAFFIC DEMAND (GBPS/KM²)

\begin{tabular}{l|lll}
\hline $\begin{array}{l}\text { Monthly } \\
\text { Demand }\end{array}$ & $\begin{array}{l}\text { Usage } \\
\text { [GB/user] }\end{array}$ & $\begin{array}{l}\text { User data } \\
\text { rate [Mbps] }\end{array}$ & $\begin{array}{l}\text { Area capacity } \\
\left.\text { G [Gbps/km }{ }^{2}\right]\end{array}$ \\
\hline Low & 30.0 & 0.25 & 16.0 \\
Moderate & 120.0 & 1.00 & 66.0 \\
High & 500.0 & 4.21 & 274.0 \\
\hline
\end{tabular}


TABLE 3: COST AND PROFITABILITY ANALYSIS OF MACRO AND METRO CELLULAR LAYOUT FOR NEW AND REUSED DEPLOYMENTS, WITH 4G AND 5G RATS, WHEN SATISFYING DENSELY POPULATED URBAN AREA OF $1.0 \mathrm{KM}^{2}$

\begin{tabular}{|c|c|c|c|c|c|c|c|c|c|c|c|c|}
\hline Deployment & \multicolumn{3}{|c|}{$4 G$ LTE-A (new sites) } & \multicolumn{3}{|c|}{$4 G$ LTE-A (sites reuse) } & \multicolumn{3}{|c|}{$5 G$ MetBS (new sites) } & \multicolumn{3}{|c|}{$5 G M e t B S$ (sites reuse) } \\
\hline GB/user/month $\left(D_{k}\right)$ & 30 & 120 & 500 & 30 & 120 & 500 & 30 & 120 & 500 & 30 & 120 & 500 \\
\hline Number of BSs & 36 & 145 & 602 & 36 & 145 & 602 & 34 & 34 & 54 & 34 & 34 & 54 \\
\hline Total CAPEX (M€) & 3.24 & 13.05 & 54.18 & 1.44 & 5.80 & 6.02 & 1.56 & 1.56 & 2.48 & 1.16 & 1.16 & 1.84 \\
\hline Annualized CAPEX (M€) & 0.59 & 2.37 & 9.85 & 0.25 & 0.98 & 4.09 & 0.25 & 0.25 & 0.39 & 0.18 & 0.18 & 0.28 \\
\hline Annual. CAPEX / GB (€) & 0.0248 & 0.0247 & 0.025 & 0.0102 & 0.0103 & 0.0102 & 0.0103 & 0.0025 & 0.00098 & 0.00729 & 0.0018 & 0.0006 \\
\hline ARPU $(€)$ & 25 & 25 & 25 & 25 & 25 & 25 & 25 & 25 & 25 & 25 & 25 & 25 \\
\hline Revenue $(€ / \mathrm{GB})$ & 0.83 & 0.21 & 0.05 & 0.83 & 0.21 & 0.05 & 0.83 & 0.21 & 0.05 & 0.83 & 0.21 & 0.05 \\
\hline 1- EBITDA Margin & $45 \%$ & $45 \%$ & $45 \%$ & $45 \%$ & $45 \%$ & $45 \%$ & $45 \%$ & $45 \%$ & $45 \%$ & $45 \%$ & $45 \%$ & $45 \%$ \\
\hline OPEX $(€ / G B)$ & 0.38 & 0.09 & 0.02 & 0.38 & 0.09 & 0.02 & 0.38 & 0.09 & 0.02 & 0.38 & 0.09 & 0.02 \\
\hline Production cost / GB (€) & 0.3998 & 0.1185 & 0.047 & 0.3852 & 0.1040 & 0.0327 & 0.3853 & 0.0963 & 0.0235 & 0.3823 & 0.0956 & 0.0232 \\
\hline EBIT or profit margin & $52.0 \%$ & $43.1 \%$ & $5.8 \%$ & $53.8 \%$ & $50.1 \%$ & $34.6 \%$ & $53.8 \%$ & $53.8 \%$ & $53.0 \%$ & $54.1 \%$ & $54.1 \%$ & $53.6 \%$ \\
\hline
\end{tabular}

Even more, if we consider the indoor deployment with the $5 \mathrm{G}$ mmW PBS we need to exclude out of (4) the $20 \mathrm{~dB}$ free space losses, and on top to consider only the inside wall attenuation of $\mathrm{W}=13.6 \mathrm{~dB}$ in line with [18]. Thus, we obtain that the new $d$ for denser indoor deployment equals 0.02 or it should be needed to deploy in total 797 $\mathrm{mmW}$ PBS sites inside the buildings to cover the area of 1 $\mathrm{km}^{2}$.

\section{Cost-Capacity Analysis and Profitability Evaluation}

In case of 4G LTE-A MaBS we consider two deployment options with an aggregation of two carriers $(2 \times 20 \mathrm{MHz})$ and with three carriers $(3 \times 20 \mathrm{MHz})$. Assuming that the carrier aggregation functionality will be part of the future 5G systems as well, we consider the aggregation of the carriers at $28 \mathrm{GHz}, 38 \mathrm{GHz}$ and $73 \mathrm{GHz}$, each with a bandwidth of $500 \mathrm{MHz}$. Thus, the capacities are doubled and tripled compared to those outlined in figure in Table 1. Due to coverage limitations in total 3,185 IEEE 802.11ad APs will be needed to cover the area of $1 \mathrm{~km}^{2}$ or around 64 per floor. Consequently, Fig. 3 depicts the resulting initial deployment costs of the single RAT network layouts to be deployed in order to satisfy a demand coming from the office areas hosting business users with a demand up to $100,000 \mathrm{~GB} / \mathrm{user} / \mathrm{month}$. The overall most cost-efficient solution is the use of the $5 \mathrm{G} \mathrm{mmW}$ PBS new sites with three frequency carriers and $500 \mathrm{MHz}$ bandwidth, whose expenditure curve above $12,000 \mathrm{~GB} / \mathrm{user} / \mathrm{month}$ shows a similar dependence on the capacity increase as the deployment with Wi-Fi IEEE 802.11ad APs.

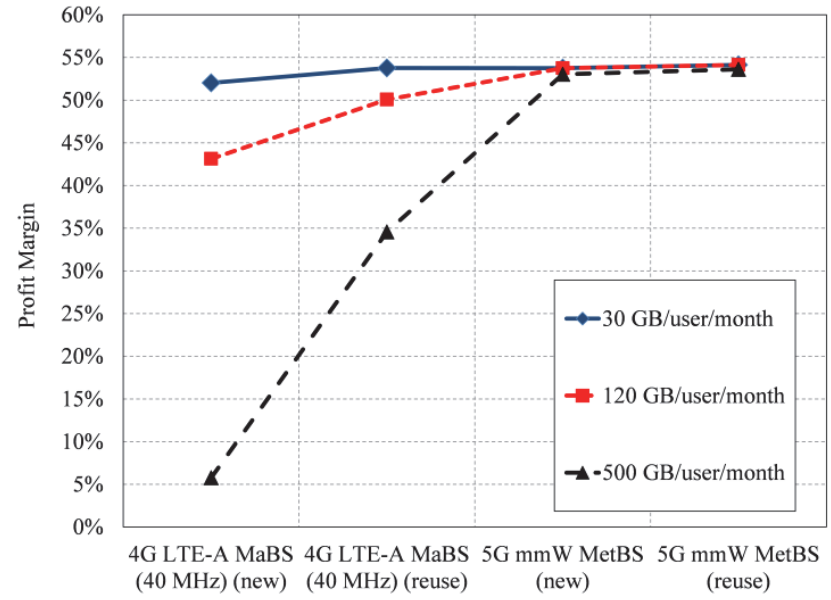

Fig. 2. Profit margin based on the annualized CAPEX.
As the area consists mostly of business users, in this case we consider a higher monthly ARPU of $50.0 €$. The 4G LTE-A MaBS deployments, seeking massive network rollout, have highly deviating profit margins and can't be considered as an appropriate option for the MNO. In the case of virtual reality office, the only deployments with the positive profit margins are the $5 \mathrm{G} \mathrm{mmW}$ PBS with three carriers (17\%) and Wi-Fi IEEE 802.11ad (16.3\%), the first being superior from capacity perspective, and the second besides a high capacity have a very low production cost.

\section{RADIO RESOURCES MANAGEMENT IN CASE OF CAPACITY OVER-PROVISIONING WITH 5G PBSS}

Here, we analyze the cases of the potential capacity overprovisioning as a result of the deployments needed to overpass the coverage limitations. Further, we use such results to consider different solutions for the application of Radio Resource Management (RRM) techniques to the cooperating MNOs. For that purpose, we assume that in reality there will be much "regular" demand from the office user with only $10 \%$ from the dimensioned 36.0 $\mathrm{TB} /$ months. In that case, the average data rate per user equals around $0.1 \mathrm{Gbps}$ and the area capacity reaches the 2,442 Gbps $/ \mathrm{km}^{2}$. Nevertheless, in case of coverage with the $5 \mathrm{G} \mathrm{mmW}$ PBS with three carriers we ensure significant overprovisioning or a 4.8 times higher capacity equal to $11,835 \mathrm{Gbps} / \mathrm{km}^{2}$.

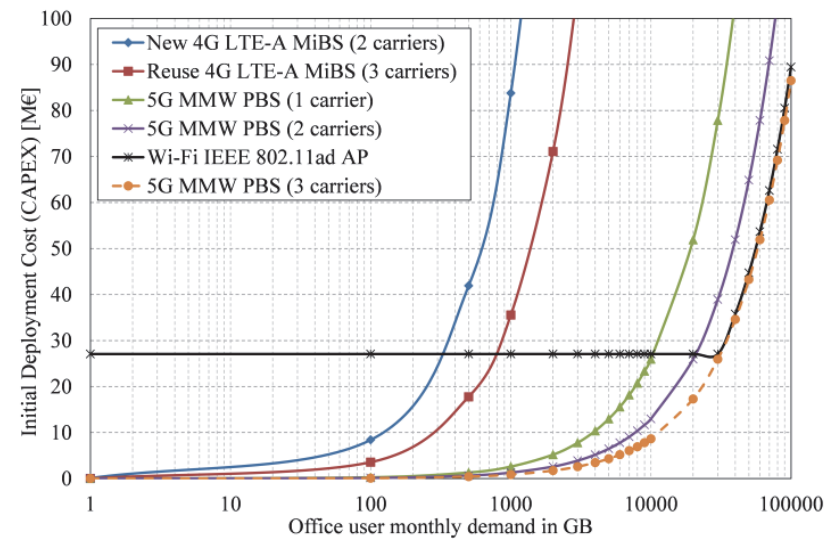

Fig. 3. Infrastructure cost for different RAT systems for office environments (logarithmic scale). 
According to [10], this overprovisioning could be exploited by ensuring guaranteed data rates to the users. Hence, based on the tele-traffic theory (the Erlang B loss formula), we could obtain the number of users being served with 1.0 Gbps guaranteed data rate with a certain service probability. For a certain Loss probability $\left(E_{\text {loss }}\right)$ and a given number of available channels per cells $C$, we calculate with the use of the "Erlang Loss Formula" [27], the offered traffic flow $O$ in Erlang and by that the average channel utilization $\eta=O / C$. In order to perform this, we assume that $1.0 \mathrm{Gbps}$ is the constant or guaranteed data rate of the user and that it corresponds to 1.0 Erlang of traffic load or it represents a capacity equal to a single channel. Consequently, the average data rate to be provided in the regular office environment of approximately $0.1 \mathrm{Gbps} / \mathrm{user}$ corresponds to a traffic load of 0.1 Erlang.

We calculate the number of "best-effort" users $N_{\text {best }}$ as a ratio between the number of available channels per cells $\mathrm{C}$ and the average data rate per user in Erlang. Finally, the number of users that could be served with a guaranteed data rate of $1.0 \mathrm{Gbps}, N_{\text {guar }}$, with a particular service probability $\left(S P=1-E_{\text {loss }}\right)$ is

$$
N_{\text {guar }}=N_{\text {best }} \frac{O_{E_{\text {loss }}}}{C}
$$

Now, considering that the cell capacity of the $5 \mathrm{G} \mathrm{mmW}$ PBS with three carriers is around 14 Gbps (or 14 channels), we could calculate the probability of certain number of users to be served with a guaranteed data rate of $1.0 \mathrm{Gbps}$, compared to the case when the average data rate is $0.1 \mathrm{Gbps}$ and could be ensured to 140 users.

The capacity of a cellular network weakens with more firm requirements on outage probability. Consequently, Fig. 4 depicts the number of served users with the guaranteed data rate of $1.0 \mathrm{Gbps}$ as a function of the total number of channels $C$ per cell with different average blocking probabilities $E_{\text {loss }} \in\{0.05,0.1,0.15,0.2\}$. It can be seen that with $80 \%, 85 \%, 90 \%$ and $95 \%$ of probability 144, 129, 114 and 97 users could be served with a constant data rate of $1.0 \mathrm{Gbps}$, respectively.

Finally, in line with [14] this approach could be used as an advanced method Radio Resource Management (RRM) functionality between MNOs that aim to share the radio resources or to jointly use a single carrier for the purpose of lowering the investment costs as compared to the case when each MNO uses a dedicated carrier.

Thus, a fixed fraction of the cell capacity can be reserved for each MNO and only one carrier utilized. So, with two MNOs equally sharing the capacity with $C_{1}=C_{2}$ $=C / 2=7.0$ channels (equally sized shares) the total capacity is reduced with $23 \%, 19 \%, 16 \%$ and $14 \%$ in case of $5 \%, 10 \%, 15 \%$ and $20 \%$ of loss probability, respectively.

For the purpose of avoiding this fixed RRM solution that will lead to a significant loss in total system capacity or, at the end, could jeopardize the cost efficiency, based on the approach elaborated in [14], we further consider a dynamical prioritization of the MNOs resource shares based on their current load. The prioritization is implemented with priority queuing in the admission control, where each connection belonging to one $\mathrm{MNO}$ should receive a priority calculated based on MNO's current load relative to their agreed minimum capacity.

Next, we briefly describe the analytics of the so called "method with non-preemptive priority queuing in admission control" that is originally introduced in [14]. So, according to [14], we consider the standard Poisson traffic model according to which the total offered load per MNO $i$ is denoted $O i$, and is defined as

$$
O_{i}=\lambda_{i} T \text {. }
$$

where $\lambda_{i}$ is the average arrival rate of new connections for MNO $i$ and $T$ is the average duration per connection. The total offered load in our case can be given by $\mathrm{O}=\mathrm{O}_{1}+\mathrm{O}_{2}$. The total number of channels per cell $C$ is still modeled as constant, or each MNO has a prioritized access to a total number of $C_{1}=C_{2}=C / 2$. Further, the priority level of each $\mathrm{MNO}, P i$, is defined as

$$
P_{i}=C_{i} / L_{i} \text {. }
$$

so that MNOs with a load $L i$ lower than the agreed minimum capacity $C i$ receives a higher priority. How effective the differentiation in blocking probability is depends on the probability that enough resources are released before the maximum allowed waiting time $T_{\max }$ is reached, after what a connection should be blocked. The performance can be evaluated by observing the MNO specific blocking probability $B_{i}$ that should be below a certain threshold $B_{\max }$ until the operator reaches its agreed minimum capacity $C$. or:

$$
B_{i} \leq B_{\max } \text { for } O_{i}<C_{i} .
$$

A connection is admitted if there is at least one channel available, that is if

$$
\sum_{i=1}^{N} L_{i}<C
$$

and blocked if no channel is released before the maximum allowed waiting time $T_{\max }$ is exceeded. In our case, we consider the total number of channels is $C=14$ for the data service, allowed queuing time $T_{\max }=15 \mathrm{~s}$, average connection time is $12 \mathrm{~s}$, and data rate is $1.0 \mathrm{Gbps}$. Table 4 summarizes the simulation results for the achieved gain relative to the case with a fixed allocation of 7 data channels.

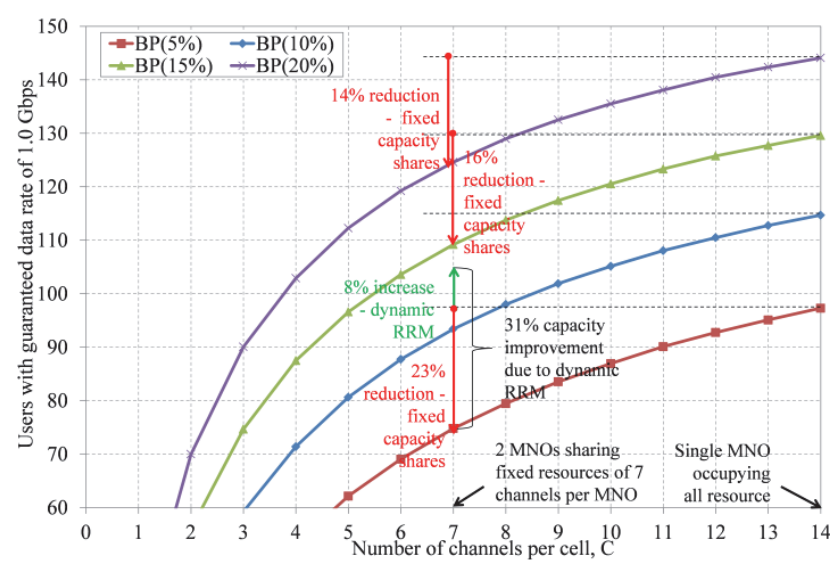

Fig. 4. Served users with the guaranteed data rate of 1.0

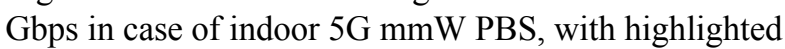
capacity changes due to particular RRM used. 
TABLE 4: CAPACITY GAIN RELATIVE TO A FIXED CHANNEL ALLOCATION WITH 5\% BLOCKING PROBABILITY

\begin{tabular}{l|cccc}
\hline Parameter & \multicolumn{4}{|c}{ Value } \\
\hline $\mathrm{O}_{2}[\mathrm{Erl}] / \mathrm{O}_{1}[\mathrm{Erl}]$ & $700 \%$ & $220 \%$ & $85 \%$ & $20 \%$ \\
Capacity gain & $-64 \%$ & $-11 \%$ & $40 \%$ & $125 \%$ \\
\hline
\end{tabular}

The blocking probability of the first $\mathrm{MNO} B_{1}$ is referred to the tolerable value of $B_{\max }=5 \%$ and for different levels of load for the other MNO $\left(\mathrm{O}_{2}\right)$ compared to the load of the evaluated MNO $\left(O_{1}\right)$. It can be seen that when the load is high for the second MNO $\mathrm{O}_{2}$ compared to the load of MNO $O_{l}$, there is a loss in capacity as compared to the reference case with 7 dedicated channels. However, with a moderate load for the second MNO, for example reaching $85 \%$ of the load of the evaluated MNO $O_{l}$, there is an increase of the carrier load of the MNO $O_{1}$ for around $40 \%$ in comparison to its load in case of the fixed channel reservation. Consequently, the average channel utilization $\eta$ will be increased from 0.53 to 0.75 , what value compared to the channel utilization in case of single MNO brings even a capacity increase of $8 \%$. Even more, if we compare this result with the result of the comparison between the channels utilizations of single only and two MNOs in case of fixed channel reservation, the result shows a significant capacity improvement of $31 \%$. This is depicted in Fig.4, where one can see the capacity gain with the "RRM method with priority queuing in admission control" in comparison to a fixed RRM share.

\section{CONCLUSION}

This paper discusses a theoretical and instructive techno-economic model developed to evaluate the costeffectiveness of the future AWA-HetNets. The results show that the future $5 \mathrm{G} \mathrm{mmW}$ system can be a way for MNOs to ensure their profit sustainable on a high level (more than 50\% profit margins). A comparable deployment option with more than $15 \%$ profit margin comes with the Wi-Fi IEEE 802.11ad, too. Also, we introduce a method to be exploited in a way to differentiate the users requiring for a guaranteed data rate of $1.0 \mathrm{Gbps}$ in case of $5 \mathrm{G}$ pico base stations deployments. Further, we analyze the cooperative resource sharing. We show that despite the RRM approach that could provide a perfect fair sharing of the available capacity (with fixed capacity shares), the cost MNOs have to pay for the fair capacity allocation is utmost likely higher than the value added due to the capacity reduction. For that purpose, we extend the analysis to the use of the resource sharing method with the use of RRM with priority queuing that dynamically adjusts to the load of the MNOs. The results show satisfactory capacity improvements of $31 \%$ when two MNOs handle comparable loads in comparison to the situation of RRM approach assuring fixed resource shares.

\section{REFERENCES}

[1] ETSI. Requirements for further advancements for Evolved Universal Terrestrial Radio Access (E-UTRA) (LTE-Advanced) (3GPP TR 36.913 version 10.0.0 Release 10). 2011.
[2] IEEE Standard 802.15.3c-2009 - for Information technology-Local and metropolitan area networks-- Amendment 2: Millimeterwave-based Alternative Physical Layer Extension. 2009.

[3] M. R. Akdeniz, et al., "Millimeter Wave Channel Modeling and Cellular Capacity Evaluation”. Cornell University Library. 2013.

[4] Z. Hang, et al., "Indoor $28 \mathrm{GHz}$ Millimeter Wave Cellular Communication Measurements for Reflection and Penetration Loss in and around Buildings in New York City". IEEE ICC. 2013.

[5] T. S. Rappaport et al., "Millimeter Wave Mobile Communications for 5G Cellular: It Will Work!"' IEEE Access, vol. 1, pp. 335-349, 2013.

[6] S. Rangan, T. S. Rappaport, E. Erkip, "Millimeter Wave Cellular Wireless Networks". arXiv:1401.2560v1 [cs.NI] 11 Jan 2014.

[7] K. Johansson, "Cost Effective Deployment Strategies for Heterogeneous Wireless Networks", Doctoral Dissertation. The Royal Institute of Technology, Stockholm, 2007.

[8] K. Johansson, A. Furuskar, P. Karlsson, and J. Zander, "Relation between base station characteristics and cost structure in cellular systems", Proceedings of IEEE PMRC, 2004.

[9] J. Markendahl, Ö. Mäkitalo, "A comparative study of deployment options, capacity and cost structure for macrocellular and femtocell networks", in Proceedings of (IOFC 2010), Istanbul, 2010.

[10] J. Markendahl, "Mobile Network Operators and Cooperation", Doctoral Dissertation. The Royal Institute of Technology, Stockholm, 2011.

[11] Z. Frias and J. Pérez, "Techno-economic analysis of femtocell deployment in long-term evolution networks", EURASIP, 2012.

[12] B. Mölleryd, J. Markendahl, O. Mäkitalo, "Mobile Broadband Expansion Calls for More Spectrum or Base Stations", European Regional ITS Conference, Copenhagen, 13-15 September 2010.

[13] R. Popescu, A. Ghanbari, J. Markendahl, "Complementing macrocell deficits with either smallcells or Wi-Fi-willingness to choose based on the cost-capacity analysis", 24th European Regional Conference of the International Telecommunication Society, Florence, Italy, 20-23 October 2013.

[14] K. Johansson, et al., "Radio resource management in roaming based multi-operator WCDMA networks". Vehicular Technology Conference, 2004. VTC 2004-Spring. 2004 IEEE 59th (Vol:4), p. 2062 - 2066, 17-19 May 2004.

[15] J. Hultell, "Cooperative and non-cooperative wireless access", Doctoral Dissertation. The Royal Institute of Technology, Stockholm, 2008.

[16] K. Johansson and A. Furuskär, "Cost efficient capacity expansion strategies using multi-access networks", In Proc. IEEE VTC, 2005.

[17] 3GPP, "Further advancements for E-UTRA physical layer aspects," TR 36.814 (release 9), 2010.

[18] F. Khan and Z. Pi, "Millimeter-wave Mobile Broadband (MMB): Unleashing 3-300GHz Spectrum," in Proc. IEEE Sarnoff Symposium, Mar. 2011.

[19] K. Johansson et al, "Modelling the cost of heterogeneous wireless access networks". Int. J. MNDI, Vol. 2, No. 1, 2007.

[20] Y. Xiao, "IEEE 802.11n: Enhancements for higher throughput in wireless LANs". IEEE Wirel Commun 12(6):82-91 2005.

[21] C. Wang and H. Wei, "IEEE 802.11n MAC Enhancement and Performance Evaluation". Mobile Networks and Applications 14, 6 (2009), 760-771, 2009

[22] E. Perahia, M. X. Gong, "Gigabit wireless LANs: An overview of IEEE 802.11ac and 802.11ad". Mobile Computing and Communications Review, 15(3), 23-33. 2011.

[23] Cisco. "Visual Networking Index: Global Mobile Data Traffic Forecast Update," 2014-2019. Feb. 2015.

[24] METIS (Mobile and wireless communications Enablers for the Twenty-twenty Information Society) Project, "Scenarios, requirements and KPIs for 5G mobile and wireless system", Document Number: ICT-317669-METIS/D1.1, 2013.

[25] "2010 Census of Population and Housing: National Capital Region". National Statistics Office of the Republic of the Philippines. Retrieved 1 July 2015.

[26] S. Tombaz et all., "Is backhaul becoming a bottleneck for green wireless access networks?". IEEE ICC. 2014.

[27] H. Leijon, Extract from the Table of the Eralng B Formula. ITU. Retrieved 1 July 2015. 